\title{
Capsule Commentary on Leiss et al., Polypharmacy Is Associated with an Increased Risk of Bleeding in Elderly Patients with Venous Thromboembolism
}

\author{
Marie-Antoinette Sevestre, MD, PhD \\ Department of Vascular Medicine, CHU Picardie, Amiens, France.
}

B leeding complications in the elderly continue to be the nightmare of anticoagulant prescribers. ${ }^{1}$ In this prospective cohort study, Leiss et al. ${ }^{2}$ investigated whether polypharmacy ( $>4$ drugs) could increase the risk of bleeding in elderly patients ( $>65$ years) who received vitamin $\mathrm{K}$ antagonists (VKA) for acute venous thromboembolism (VTE). After adjusting to known bleeding risk factors and vitamin $\mathrm{K}$ treatment duration, patients with polypharmacy $(49.8 \%)$ had a significant higher incidence of major bleeding events (sub-hazard ratio 1.83) and clinically relevant non-major bleeding (sub-hazard ratio 1.60) than patients without polypharmacy. The authors conclude that polypharmacy is a risk factor for bleeding in elderly patients treated with VKA for VTE.

This study gives important clues to lower bleeding risk in patients treated with anticoagulants, such as: avoiding the use of VKA drug inductors, reducing the risk of falls by stopping sedative drugs, and avoiding a harmful concomitant prescription of aspirin or nonsteroidal anti-inflammatory drugs (NSAIDs). The authors also suggest that polypharmacy is a good marker of disease burden and that INR control might not be optimal in elderly patients with polypharmacy. This study raises two important questions:

- Considering that trials testing direct oral anticoagulants have shown similar efficacy treating VTE and preventing stroke in the case of atrial fibrillation with less bleeding complications than VKA, how should we use them in the elderly?

- How long do we need to treat elderly patients for VTE and how do we calculate the benefit/risk ratio?

Answers will be given by longitudinal studies of elderly patients treated with direct oral anticoagulants. Meta analyses from randomized trials show encouraging results, but need to be tested in "real life conditions."

When a patient is treated for VTE during three months, the decision to stop anticoagulants or to continue is driven by the risk of VTE recurrence balanced by the risk of bleeding, calculated through dedicated scores. Finding the molecule and dosage capable of reducing both cardiovascular risk and VTE recurrence with the fewest bleeding complications is the challenge of the near future.

Conflict of Interest: The author has no conflict with any material of this commentary.

Corresponding Author: Marie-Antoinette Sevestre, $\mathrm{MD}, \mathrm{PhD} ; \mathrm{De}$ partment of Vascular Medicine, CHU Picardie, Amiens, France (e-mail: Sevestre.marie-antoinette@chu-amiens.fr).

\section{REFERENCES}

1. Landefeld CS, Beyth RJ. Anticoagulant-related bleeding: clinical epidemiology, prediction and prevention. Am J Med. 1993;95(3):315-28.

2. Leiss W, Mean M, Limacher A, Righini M, Jaeger $\mathbf{K}$, Beer $\mathbf{H J}$, Osterwalder J, Frauchiger B, Matter CM, Kucher N, Angelillo-Scherrer A, Cornuz J, Banyai M, Lämmle B, Husmann M, Egloff M, Aschwanden M, Rodondi N, Aujesky D. Polypharmacy is associated with an increased risk of bleeding in elderly patients with venous thromboembolism. $\mathrm{J}$ Gen Intern Med 2014 (Article SPI\#2993).

3. Sardar P, Chatterjee S, Chaudhari S, Lip GY. New oral anticoagulants in elderly adults: evidence from a meta-analysis of randomized trials. J Am Geriatr Soc. 2014;62(5):857-64. 